\title{
EXACT BOUNDARY CONTROLLABILITY OF UNSTEADY FLOWS IN A TREE-LIKE NETWORK OF OPEN CANALS*
}

\author{
TA-TSIEN LI ${ }^{\dagger}$ AND BOPENG RAO $\ddagger$
}

\begin{abstract}
In this paper we establish the exact boundary controllability of unsteady flows in a tree-like network of open canals with general topology.

Key words. Exact boundary controllability, Saint-Venant system, tree-like network of open canals
\end{abstract}

AMS subject classifications. 93B05, 35B37, 93C20, 35L50

1. Introduction. The goal of this paper is to establish the exact boundary controllability of unsteady flows in a tree-like network of open canals.

The one-dimensional mathematical model of unsteady flows in an open canal was given by Saint-Venant system [1], which has been frequently used by hydraulic engineers in their practice (see also [2]-[7]). Using the theory on the semi-global $C^{1}$ solution and the exact boundary controllability for quasilinear hyperbolic systems (cf [10-15]), the exact boundary controllability of unsteady flows in both a single open canal and a star-like network of open canals was obtained in [8]. Later on, the exact boundary controllability of unsteady flows in a string-like network of open canals was established in [9].

In this paper we will generalize the previous results to a tree-like network of open canals with general topology. For this purpose, we will serve the total flux at the multiple node as an interface control. By means of a method different from that in [8], we establish another type of result on the exact boundary controllability of unsteady flows in a star-like network of open canals. Based on this new result and the results obtained in [8-9] on the exact boundary controllability of unsteady flows for a single open canal, a star-like network and a string-like network of open canals, we establish the exact boundary controllability of unsteady flows in a tree-like network of open canals with general topology.

This paper is organized as follows. In $\S 2$ we give the statement of the exact boundary controllability of unsteady flows in a network of open canals and show the main idea of realizing it. In $\S 3$, we recall some results given in [8-9] on the exact boundary controllability of unsteady flows in a network of open canals with simpler topology. In $\S 4$, a fundamental Lemma on the continuation of piecewise $C^{1}$ solution in a star-like network of open canals is established. Then, combining the results in $\S \S 3-4$, we establish in $\S 5$ the exact boundary controllability of unsteady flows in a general tree-like network of open canals. Finally, some remarks are given in $\S 6$.

2. Statement on the exact boundary controllability of unsteady flows in a network of open canals. We consider unsteady flows in a network composed of $N$ open canals. Let $a_{i 0}$ and $a_{i 1}$ be the $x$-coordinates of two ends of the $i$-canal and

\footnotetext{
*Received July 21, 2004; accepted for publication November 14, 2004.

†School of Mathematical Sciences, Fudan University, Shanghai 200433, China (dqli@fudan. edu.cn).

¥Institut de Recherche Mathématique Avancée, Université Louis Pasteur de Strasbourg, 67084 Strasbourg, France (rao@math.u-strasbg.fr).
} 
$L_{i}=a_{i 1}-a_{i 0}$ its length. Suppose that there is no friction and the canal is horizontal and cylindrical, the corresponding Saint-Venant system can be written as (cf. [2], [8]-[9])

$$
\left\{\begin{array}{l}
\frac{\partial A_{i}}{\partial t}+\frac{\partial\left(A_{i} V_{i}\right)}{\partial x}=0, \\
\frac{\partial V_{i}}{\partial t}+\frac{\partial S_{i}}{\partial x}=0,
\end{array} \quad t \geq 0, \quad a_{i 0} \leq x \leq a_{i 1}(i=1, \cdots, N)\right.
$$

where, for the $i$-th canal, $A_{i}=A_{i}(t, x)$ stands for the area of the cross section at $x$ occupied by the water at time $t, V_{i}=V_{i}(t, x)$ the average velocity over the cross section and

$$
S_{i}=\frac{1}{2} V_{i}^{2}+g h_{i}\left(A_{i}\right)+g Y_{i b},
$$

where $g$ is the gravity constant, constant $Y_{i b}$ denotes the altitude of the bed and

$$
h_{i}=h_{i}\left(A_{i}\right)
$$

is the depth of the water, $h_{i}\left(A_{i}\right)$ being a suitably smooth function of $A_{i}$ such that

$$
h_{i}^{\prime}\left(A_{i}\right)>0 .
$$

The initial condition is

$$
t=0: \quad\left(A_{i}, V_{i}\right)=\left(A_{i 0}(x), V_{i 0}(x)\right), \quad a_{i 0} \leq x \leq a_{i 1}(i=1, \cdots, N) .
$$

When $a_{i 0}\left(\right.$ resp. $a_{i 1}$ )is a simple node, we have the flux boundary condition:

$$
x=a_{i 0}: \quad A_{i} V_{i}=q_{i 0}(t) \quad\left(\text { resp. } x=a_{i 1}: \quad A_{i} V_{i}=q_{i 1}(t)\right) .
$$

While, when $a_{i 0}$ (resp. $\left.a_{i 1}\right)$ is a multiple node, we denote by $\mathcal{J}_{i 0}$ (resp. $\left.\mathcal{J}_{i 1}\right)$ the set of indices corresponding to all the canals jointed at $a_{i 0}$ (resp. $a_{i 1}$ ). At $a_{i 0}$ (resp. $a_{i 1}$ ) we have (cf. [2],[8])the energy-type interface conditions

$$
S_{i}=S_{j}, \quad \forall j \in \mathcal{J}_{i 0} \quad\left(\text { resp. } \forall j \in \mathcal{J}_{i 1}\right)
$$

and the total flux interface condition

$$
\sum_{j \in \mathcal{J}_{i 0}} \pm A_{j} V_{j}=Q_{i 0}(t) \quad\left(\text { resp. } \sum_{j \in \mathcal{J}_{i 1}} \pm A_{j} V_{j}=Q_{i 1}(t)\right) .
$$

An equilibrium state $\left(A_{i}, V_{i}\right)=\left(\bar{A}_{i}, \bar{V}_{i}\right)$ with $\bar{A}_{i}>0(i=1, \cdots N)$ is subcritical if

$$
\left\{\begin{array}{l}
\lambda_{i 0}=\bar{V}_{i}-\sqrt{g \bar{A}_{i} h_{i}^{\prime}\left(\bar{A}_{i}\right)}<0, \\
\mu_{i 0}=\bar{V}_{i}+\sqrt{g \bar{A}_{i} h_{i}^{\prime}\left(\bar{A}_{i}\right)}>0,
\end{array} \quad(i=1, \cdots, N) .\right.
$$


For the purpose of the exact boundary controllability of unsteady flows in a network of open canals, we want to find a time $T>0$ such that for any given initial data $\left(A_{i}, V_{i}\right)=\left(A_{i 0}(x), V_{i 0}(x)\right)$ and final data $\left(A_{i}, V_{i}\right)=\left(A_{i T}(x), V_{i T}(x)\right)(i=1, \cdots N)$ in a $C^{1}$ neighbourhood of a given subcritical equilibrium state $\left(\bar{A}_{i}, \bar{V}_{i}\right)(i=1, \cdots N)$, there exist suitable flux boundary controls at some simple nodes and suitable total flux interface controls at some multiple nodes, such that the corresponding mixed initialboundary value problem for Saint-Venant system (2.1) with the initial data (2.5), the corresponding boundary conditions (2.6) and the interface conditions (2.7)-(2.8) admits a unique piecewise $C^{1}$ solution on the time interval $0 \leq t \leq T$, which satisfies exactly the final condition

$$
t=T: \quad\left(A_{i}, V_{i}\right)=\left(A_{i T}(x), V_{i T}(x)\right), \quad a_{i 0} \leq x \leq a_{i 1}(i=1, \cdots, N) .
$$

To this end, it suffices to construct a piecewise $C^{1}$ solution to Saint-Venant system (2.1) on the time interval $0 \leq t \leq T$, which satisfies the initial condition (2.5), the final condition (2.10), all the energy-type interface conditions (2.7) and a part of flux boundary conditions (2.6) and total flux interface conditions (2.8). Once this solution is obtained, we put it into the rest of flux boundary conditions (2.6) and total flux interface conditions (2.8) to get the desired flux boundary controls $q_{i 0}(t)$ (resp. $q_{i 1}(t)$ ) and total flux interface controls $Q_{i 0}(t)$ (resp. $Q_{i 1}(t)$ ), which realize the exact boundary controllability for the Saint-Venant system.

To construct this kind of piecewise $C^{1}$ solution is a nonstandard problem which can be regarded as an inverse problem. There is no uniqueness in general. However we can get this solution in a stable way, i.e., by solving several well-posed problems. The main principle in the resolution consists in making the number of controls or the controllability time $T$ as small as possible. However, for a smaller number of controls, we need a larger controllability time, and vise versa.

3. Construction of piecewise $C^{1}$ solution in a network of canals with simpler topology. In this section, we recall some results given in [8-9], which will be useful in what follows.

1). Consider a single canal with flux boundary conditions at one end. If

$$
T>2 L \max \left\{-\frac{1}{\lambda_{0}}, \frac{1}{\mu_{0}}\right\}
$$

where $\lambda_{0}$ and $\mu_{0}$ are given by (2.9) and $L=a_{1}-a_{0}$ is the length of the canal, we can construct a $C^{1}$ solution on the single canal, such that it satisfies any given initial and final conditions in a $C^{1}$ neighbourhood of a given subcritical equilibrium state $(\bar{A}, \bar{V})$ as well as the the flux boundary condition on one end (see [8] for the detail). We illustrate this procedure by the following figure:

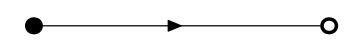

FIG. 3.1. A single canal

Here a bold point "•" denotes the starting end on which the flux boundary condition is satisfied, while a hollow point "o" denotes the arriving end, on which the value of solution is determined by the procedure of resolution, and the arrow denotes the direction along which we solve the problem. 
2 ). By rescaling and folding all corresponding $x$-interval to the interval $0 \leq x \leq 1$, the previous method can be applied to a star-like network of $N$ open canals (see [8] for the detail). Let $T$ satisfy

$$
T>2 \max _{1 \leq i \leq N}\left(L_{i} \max \left\{-\frac{1}{\lambda_{i 0}}, \frac{1}{\mu_{i 0}}\right\}\right),
$$

where $\lambda_{i 0}$ and $\mu_{i 0}$ are given by (2.9) and $L_{i}=a_{i 1}-a_{i 0}$ is the length of the $i$-th canal $(i=1, \cdots N)$. Then, by solving the problem from the joint end to all simple nodes, we can get a piecewise $C^{1}$ solution which satisfies any given initial and final conditions in a piecewise $C^{1}$ neighbourhood of a given subcritical equilibrium $\left(\bar{A}_{i}, \bar{V}_{i}\right)(i=1, \cdots N)$ as well as the interface conditions (2.7)-(2.8) at the joint end (the multiple node). We illustrate this procedure by the following figure:

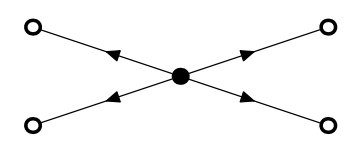

FIG. 3.2. A star-like network of canals

Here all simple nodes are the arriving ends "o" on which the solution is determined by the procedure of resolution. The joint node is the starting end "•" on which all the interface conditions (2.7)-(2.8) are satisfied. The procedure of resolution is indicated by the arrows: from "•" to "०".

3). For a string-like network of $N$ open canals, if

$$
T>2 \sum_{i=1}^{N} L_{i} \max \left\{-\frac{1}{\lambda_{i 0}}, \frac{1}{\mu_{i 0}}\right\},
$$

where $\lambda_{i 0}$ and $\mu_{i 0}$ are given by (2.9) and $L_{i}=a_{i 1}-a_{i 0}$ is the length of $i$-th canal $(i=1, \cdots N)$, by solving the problem from one simple node "•" to another simple node "o", indicated by Figure 3.3, we can still get a piecewise $C^{1}$ solution which satisfies any given initial and final conditions in a piecewise $C^{1}$ neighbourhood of a given subcritical equilibrium $\left(\bar{A}_{i}, \bar{V}_{i}\right)(i=1, \cdots N)$ as well as the flux boundary condition on one simple node and the interface conditions (2.7)-(2.8) on all the multiple nodes denoted by "." (see [9] for the detail). This situation is similar to the case shown by Figure 3.1 for a single canal, however, the controllability time should be much larger.

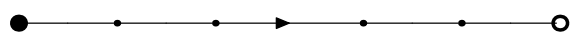

FIG. 3.3. A string-like network of canals

4). Based on the results given in 2) and 3), if $T$ is large enough (bigger than the maximum of the exact controllability time corresponding to each string-like subnetwork), similarly we can get a piecewise $C^{1}$ solution for a star-like network of stringlike sub-networks, which satisfies any given initial and final conditions in a piecewise $C^{1}$ neighbourhood of a given subcritical equilibrium as well as the interface conditions (2.7)-(2.8) on each multiple node. We illustrate this procedure by the following figure: 


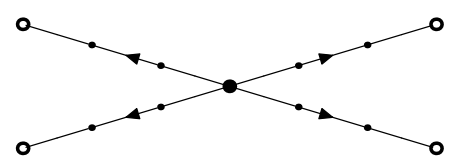

FIG. 3.4. A star-like network of string-like sub-networks

Here the joint node of all the string-like sub-networks is the starting end "•", all the simple nodes are the arriving end "o", while all the joint nodes in the stringlike sub-networks are denoted by "." on which the interface conditions (2.7)-(2.8)are satisfied.

4. Fundamental Lemma on the continuation of piecewise $C^{1}$ solution. In order to get a desired piecewise $C^{1}$ solution for a network of $N$ open canals with general topology, we should extend the piecewise $C^{1}$ solution on a network of open canals with simpler topology shown in the previous section. To this end, we now give another way to construct a piecewise $C^{1}$ solution for a star-like network of $N$ open canals by assuming that a $C^{1}$ solution satisfying any given initial and final conditions in a $C^{1}$ neighbourhood of a corresponding subcritical equilibrium is obtained on one canal, and then solving the problem from the joint end to other simple ends respectively, which is illustrated in the following figure:

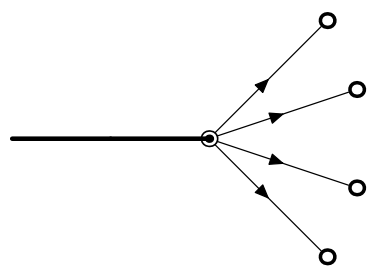

FIG. 4.1 A star-like network of canals

Let $\left(A_{1}, V_{1}\right)$ be a known $C^{1}$ solution on the first canal denoted by "__ ". In order to construct the desired $C^{1}$ solution on other canals in the star-like network, we serve the energy-like interface condition as a boundary condition at the joint node $x=0$ for each corresponding canal. Thus the piecewise $C^{1}$ solution constructed satisfies only the energy-like interface conditions (2.7), while the total flux interface condition (2.8) is no longer satisfied at the joint node which is then denoted by "๑". More precisely, we have the following result.

Lemma 4.1. Let $\left(A_{i}, V_{i}\right)=\left(\bar{A}_{i}, \bar{V}_{i}\right)(i=1, \cdots, N)$ be a subcritical equilibrium state. Suppose that

$$
T>2 \max _{i=2, \cdots, N}\left\{L_{i} \max \left(-\frac{1}{\lambda_{i 0}}, \frac{1}{\mu_{i 0}}\right)\right\}
$$

where $\lambda_{i 0}$ and $\mu_{i 0}(i=2, \cdots, N)$ are still given by (2.9). Let $\left(A_{i 0}(x), V_{i 0}(x)\right)$ and $\left(A_{i T}(x), V_{i T}(x)\right)(i=1, \cdots, N)$ be any given initial state and final state with small $C^{1}$ norms $\sum_{i=1}^{n}\left\|\left(A_{i 0}(\cdot)-\bar{A}_{i}, V_{i 0}(\cdot)-\bar{V}_{i}\right)\right\|_{C^{1}\left[0, L_{i}\right]}$ and $\sum_{i=1}^{n}\left\|\left(A_{i T}(\cdot)-\bar{A}_{i}, V_{i T}(\cdot)-\bar{V}_{i}\right)\right\|_{C^{1}\left[0, L_{i}\right]}$, which satisfy the conditions of piecewise $C^{1}$ compatibility with the energy-like interface conditions $(2.7)$ at the points $(t, x)=(0,0)$ and $(T, 0)$ respectively. 
Let

$$
R_{i}(T)=\left\{(t, x) \mid 0 \leq t \leq T, \quad 0 \leq x \leq L_{i}\right\} \quad(i=1, \cdots, N)
$$

Assume that we have already determined the solution $\left(A_{1}, V_{1}\right)$ with small $C^{1}$ norm $\left\|\left(A_{1}-\bar{A}_{1}, V_{1}-\bar{V}_{1}\right)\right\|_{C^{1}\left[R_{1}(T)\right]}$, which satisfies the initial condition $(2.5)$ and the final condition (2.10) for $i=1$. Then there exists a piecewise $C^{1}$ solution $\left(A_{i}, V_{i}\right)(i=$ $1, \cdots, N)$ on the domain $\cup_{i=1}^{N} R_{i}(T)$ with small piecewise $C^{1}$ norm $\sum_{i=1}^{n} \|\left(A_{i}-\bar{A}_{i}, V_{i}-\right.$ $\left.\bar{V}_{i}\right) \|_{C^{1}\left[R_{i}(T)\right]}$, which satisfies the initial condition (2.5), the final condition (2.10) and the energy-like interface conditions (2.7) at the joint node $x=0$.

Proof. For $i=1, \cdots, N$, introducing the Riemann invariants $r_{i}$ and $s_{i}$ as follows:

$$
\left\{\begin{array}{l}
2 r_{i}=V_{i}-\bar{V}_{i}-G_{i}\left(A_{i}\right), \\
2 s_{i}=V_{i}-\bar{V}_{i}+G_{i}\left(A_{i}\right),
\end{array}\right.
$$

where

$$
G_{i}\left(A_{i}\right)=\int_{\bar{A}_{i}}^{A_{i}} \sqrt{\frac{g h_{i}^{\prime}\left(A_{i}\right)}{A_{i}}} d A_{i} .
$$

Let $H_{i}$ be the inverse function of $G_{i}\left(A_{i}\right)$. We have

$$
\left\{\begin{array}{l}
V_{i}=r_{i}+s_{i}+\bar{V}_{i} \\
A_{i}=H_{i}\left(s_{i}-r_{i}\right)
\end{array}\right.
$$

and

$$
H_{i}^{\prime}\left(s_{i}-r_{i}\right)=\sqrt{\frac{A_{i}}{g h_{i}^{\prime}\left(A_{i}\right)}}>0
$$

Since $\left(A_{i}, V_{i}\right)=\left(\bar{A}_{i}, \bar{V}_{i}\right)$ correspond to $\left(r_{i}, s_{i}\right)=(0,0)$, we get

$$
H_{i}(0)=\bar{A}_{i}, \quad H_{i}^{\prime}(0)=\sqrt{\frac{\bar{A}_{i}}{g h_{i}^{\prime}\left(\bar{A}_{i}\right)}}>0 .
$$

Taking $\left(r_{i}, s_{i}\right)(i=1, \cdots, N)$ as new unknown variables, system (2.1) can be reduced to the following system of diagonal form:

$$
\left\{\begin{array}{l}
\frac{\partial r_{i}}{\partial t}+\lambda_{i} \frac{\partial r_{i}}{\partial x}=0, \\
\frac{\partial s_{i}}{\partial t}+\mu_{i} \frac{\partial s_{i}}{\partial x}=0,
\end{array} \quad(i=1, \cdots N)\right.
$$

where

$$
\left\{\begin{array}{l}
\lambda_{i}=V_{i}-\sqrt{g A_{i} h_{i}^{\prime}\left(A_{i}\right)}<0, \\
\mu_{i}=V_{i}+\sqrt{g A_{i} h_{i}^{\prime}\left(A_{i}\right)}>0,
\end{array} \quad(i=1, \cdots N) .\right.
$$


The energy-type interface conditions (2.7) now become

$$
\begin{aligned}
x=0: \quad & \frac{1}{2}\left(r_{i}+s_{i}+\bar{V}_{i}\right)^{2}+g h_{i}\left(H_{i}\left(s_{i}-r_{i}\right)\right)+g y_{b i} \\
= & \frac{1}{2}\left(r_{1}+s_{1}+\bar{V}_{i}\right)^{2}+g h_{1}\left(H_{1}\left(s_{1}-r_{1}\right)\right)+g y_{b 1} \\
& (i=2, \cdots N) .
\end{aligned}
$$

For each $i=2, \cdots, N$, let

$$
\begin{aligned}
P_{i}\left(r_{i}, s_{i}, r_{1}, s_{1}\right)= & \frac{1}{2}\left(r_{i}+s_{i}+\bar{V}_{i}\right)^{2}+g h_{i}\left(H_{i}\left(s_{i}-r_{i}\right)\right)+g y_{b i} \\
& -\left(\frac{1}{2}\left(r_{1}+s_{1}+\bar{V}_{1}\right)^{2}+g h_{i}\left(H_{1}\left(s_{1}-r_{1}\right)\right)+g y_{b 1}\right) .
\end{aligned}
$$

Since the state $\left(A_{k}, V_{k}\right)=\left(\bar{A}_{k}, \bar{V}_{k}\right)$ corresponds to $\left(r_{k}, s_{k}\right)=(0,0)$ for $k=1, \cdots N$, condition (4.10) implies

$$
P_{i}(0,0,0,0)=0
$$

On the other hand, a straightforward computation gives

$$
\frac{\partial P_{i}}{\partial s_{i}}(0,0,0,0)=\bar{V}_{i}+\sqrt{g \bar{A}_{i} h_{i}\left(\bar{A}_{i}\right)}>0 .
$$

Then, by the implicit function theorem, we can find a $C^{1}$ function $f_{i}$ in a neighbourhood of $\left(r_{i}, r_{1}, s_{1}\right)=(0,0,0)$ such that $(4.10)$ can be equivalently rewritten as

$$
s_{i}=f_{i}\left(r_{i}, r_{1}, s_{1}\right)
$$

with $f_{i}(0,0,0)=0$. Since the solution $\left(r_{1}, s_{1}\right)$ is known, putting the values $r_{1}(t, 0)$ and $s_{1}(t, 0)$ into (4.14) and noting $f_{i}(0,0,0)=0,(4.14)$ can be equivalently rewritten as

$$
s_{i}=G_{i}\left(t, r_{i}\right)+H_{i}(t)
$$

where $G_{i}$ and $H_{i}$ are $C^{1}$ functions such that

$$
G_{i}(t, 0) \equiv 0
$$

and

$$
\left\|H_{i}\right\|_{C^{1}[0, T]} \text { is small enough. }
$$

Similarly, for each $i=2, \cdots, N$, we have

$$
\frac{\partial P_{i}}{\partial r_{i}}(0,0,0,0)=\bar{V}_{i}-\sqrt{g \bar{A}_{i} h_{i}\left(\bar{A}_{i}\right)}<0 .
$$

Then, in a neighbourhood of $\left(r_{i}, s_{i}, r_{1}, s_{1}\right)=(0,0,0,0)$, condition (4.10) can be equivalently rewritten as

$$
r_{i}=\bar{G}_{i}\left(t, s_{i}\right)+\bar{H}_{i}(t),
$$


where $\bar{G}_{i}$ and $\bar{H}_{i}$ are $C^{1}$ functions such that

$$
\bar{G}_{i}(t, 0) \equiv 0
$$

and

$$
\left\|\bar{H}_{i}\right\|_{C^{1}[0, T]} \quad \text { is small enough. }
$$

Moreover, under the assumption of Lemma 4.1, it is easy to see that the conditions of $C^{1}$ compatibility with (4.15)(resp.(4.19)) are still satisfied at the point $(t, x)=(0,0)$ (resp. $(T, 0))$. Then, applying the results given in [13] and [8] to system (4.8) for the $i$-th canal, we get the solution $\left(r_{i}, s_{i}\right)$, i.e., $\left(A_{i}, V_{i}\right)$ for each $i=2, \cdots, N$, which, together with $\left(A_{1}, V_{1}\right)$, satisfy the initial condition (2.5), the final condition (2.10) and the energy-type interface conditions (2.7) at $x=0$. The proof is then complete.

REMARK 4.1. Lemma 4.1 is still valid for a star-like network of string-like subnetworks, when a piecewise $C^{1}$ solution is known on one string-like sub-network and $T>0$ is suitably large.

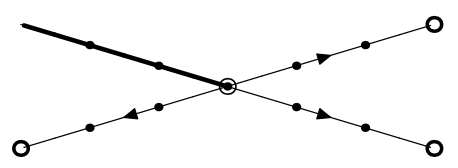

FIG. 4.2. A star-like network of string-like sub-networks

5. Exact boundary controllability of unsteady flows in a tree-like network of open canals. A network is called to have a tree-like configuration, if any two nodes in the network can be connected by a unique path of canals. In other words, a tree-like network is a connected network without loop. In what follows, for $T>0$ suitably large, taking the results given in $\S 3$ as the first step, we will successively use Lemma 4.1 or Remark 4.1 to get a desired piecewise $C^{1}$ solution on the whole network, which implies the exact boundary controllability for a tree-like network of open canals.

Arbitrarily choosing a node as the starting end, we construct a desired piecewise $C^{1}$ solution as follows.

Case A. When the starting end $O_{k}$ is a simple node, on which the boundary flux $q_{k}$ with small $C^{1}$ norm $\left\|q_{k}(\cdot)-\bar{A}_{k} \bar{V}_{k}\right\|_{C^{1}[0, T]}$ is given, we assume that the initial data and the final data satisfy the conditions of $C^{1}$ compatibility with the flux boundary condition (2.6) at $O_{k}$. For $T>0$ suitably large, by the procedure illustrated in Figure 3.1 , we can get a $C^{1}$ solution of system (2.1) on the canal with the end $O_{k}$ (another end of this canal is denoted by $O_{l}$ ), which satisfies the corresponding initial condition (2.5) and the final condition (2.10) on the canal and the flux boundary condition (2.6) at the simple node $O_{k}$. This procedure can be shown in the following figure:

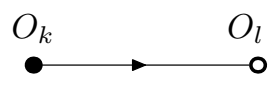

FIG. 5.1. Starting from a simple node

Still for $T>0$ suitably large, applying Lemma 4.1 illustrated in Figure 4.1 to the local star-like network of open canals jointed at the multiple node $O_{l}$, we get a 
piecewise $C^{1}$ solution which satisfies the corresponding initial condition (2.5) and the final condition (2.10) on this local star-like network, the flux boundary condition (2.6) at the simple node $O_{k}$, and the energy-like interface conditions (2.7) at the multiple node $O_{l}$. We illustrate this procedure by the following figure:

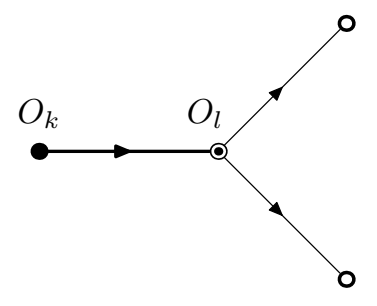

FIG. 5.2. Extension to a local star-like network

Then, repeating the procedure given by Lemma 4.1, we can construct a piecewise $C^{1}$ solution on the whole network of canals, which satisfies the initial condition (2.5) and the final condition (2.10) on the whole network, the boundary condition (2.6) at the starting end $O_{k}$ and the energy-like interface conditions (2.7) at all multiple nodes. Finally, we substitute this solution in (2.8) to get the total flux interface controls $Q_{i}(t)$ for all multiple nodes, and in (2.6) to get the flux boundary controls $q_{i}(t)$ for all simple nodes except the starting end $O_{k}$. We illustrate this procedure by the following figure:

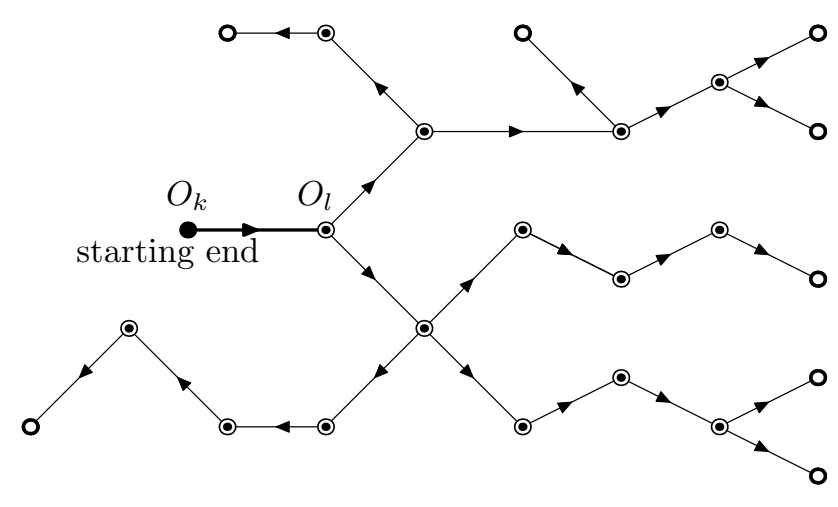

FIG. 5.3. Exact controllability starting from a simple node (23 controls for 23 canals)

Case B. When the starting end $O_{l}$ is a multiple node, on which the total flux $Q_{l}$ with small norm $\left\|Q_{l}(\cdot)-\sum_{j \in \mathcal{J}_{l}} \pm \bar{A}_{j} \bar{V}_{j}\right\|_{C^{1}[0, T]}$ is given, we assume furthermore that the initial data and the final data also satisfy the conditions of piecewise $C^{1}$ compatibility with the total flux interface condition (2.8) at the multiple node $O_{l}$. For $T>0$ suitably large, by the procedure illustrated in Figure 3.2, we get a piecewise $C^{1}$ solution for the local star-like network jointed at the multiple node $O_{l}$, which satisfies the corresponding initial condition (2.5) and the final condition (2.10) on the whole local star-like network, the interface conditions $(2.7)$ and (2.8) at multiple node $O_{l}$. We illustrate this procedure by the following figure. 


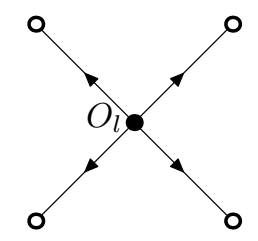

FIG. 5.4. Starting from a multiple node

Then repeating the procedure given by Lemma 4.1 as shown in the previous situation, we finally get a piecewise $C^{1}$ solution for the whole network of canals, which satisfies the initial condition (2.5) and the final condition (2.10) on the whole network, the total flux interface condition (2.8) at the starting multiple node $O_{l}$ and the energy-type interface conditions (2.7) at all multiple nodes. Thus, in a similar way, we can define the flux boundary controls $q_{i}(t)$ for all simple nodes, and the total flux controls $Q_{i}(t)$ for all multiple nodes except the starting end $O_{l}$, respectively. We illustrate this procedure by the following figure:

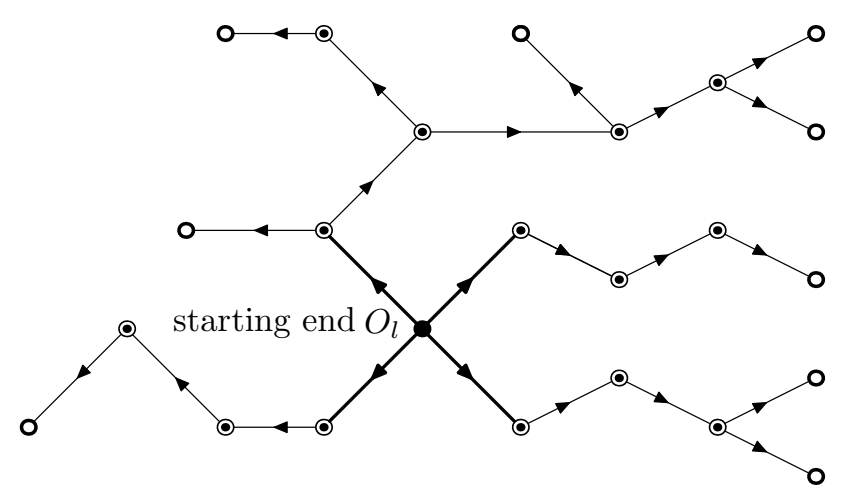

FIG. 5.5. Exact controllability starting from a multiple node (23 controls for 23 canals)

Case C. If the tree-like network contains string-like sub-networks, we can use the procedure shown in Figure 3.3 or Figure 3.4 as the first step and then apply repeatedly Remark 4.1 to get a desired $C^{1}$ piecewise solution for the whole network of the canals (see Figures 5.6 and 5.7). In this way we can reduce the number of controls, however, the controllability time should be correspondingly enlarged (cf. (3.4)). 


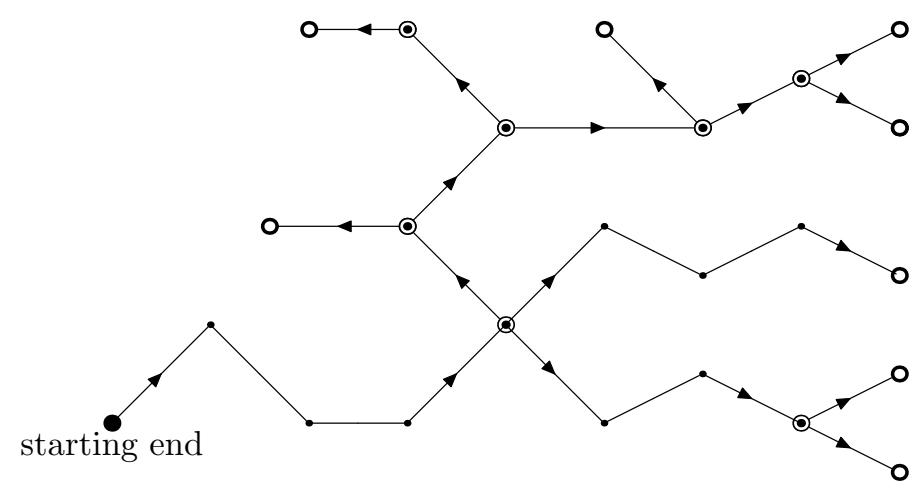

FIG. 5.6. Exact controllability starting from a simple node

for a tree-like network containing string-like sub-networks (15 controls for 23 canals)

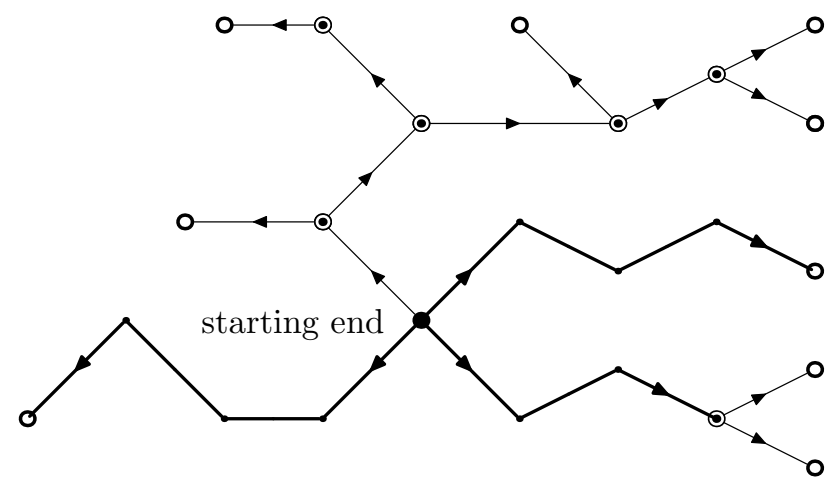

FIG. 5.7. Exact controllability starting from a multiple node

for a tree-like network containing string-like sub-networks (15 controls for 23 canals)

6. Some remarks. 1). The exact controllability time obtained in each case is optimal.

2). The flux boundary condition (2.6) can be replaced by the water level boundary condition

$$
x=a_{i 0}: \quad A_{i}=q_{i 0}(t) .
$$

Accordingly, the energy-like interface conditions (2.7) can be also replaced by the water level interface conditions

$$
h_{i}\left(A_{i}\right)+Y_{i b}=h_{j}\left(A_{j}\right)+Y_{j b}, \quad \forall j \in \mathcal{J}_{i 0} .
$$

3). The flux boundary condition (2.6) can be replaced by the following boundary condition of underflow gate (cf.[7])

$$
x=a_{i 0}: \quad A_{i} V_{i}\left|A_{i} V_{i}\right|=2 g u_{i 0}^{2}\left(y_{i 0}-h_{i}\left(A_{i}\right)\right),
$$


where $y_{i 0}$ is the water level outside the $i$-th canal and $u_{i 0}$ is the height of gate opening at $x=a_{i 0}$.

Accordingly, on each intermediate joint end $a_{i 0}$ of a string-like sub-network, the interface conditions (2.7)-(2.8) can be also replaced by the interface conditions of underflow gate (cf.[7])

$$
A_{i-1} V_{i-1}\left|A_{i-1} V_{i-1}\right|=A_{i} V_{i}\left|A_{i} V_{i}\right|=2 g u_{i 0}^{2}\left(h_{i-1}\left(A_{i-1}\right)-h_{i}\left(A_{i}\right)\right) .
$$

4). When one considers the friction in a network of open canals, Saint-Venant system (2.1) becomes

$$
\left\{\begin{array}{l}
\frac{\partial A_{i}}{\partial t}+\frac{\partial\left(A_{i} V_{i}\right)}{\partial x}=0, \\
\frac{\partial V_{i}}{\partial t}+\frac{\partial S_{i}}{\partial x}+F_{i}\left(A_{i}, V_{i}\right)=0
\end{array} \quad t \geq 0, \quad a_{i 0} \leq x \leq a_{i 1}(i=1, \cdots, N)\right.
$$

with $F_{i}\left(A_{i}, 0\right) \equiv 0$ and $V_{i} F_{i}\left(A_{i}, V_{i}\right) \geq 0$. For any given equilibrium state $\left(A_{i}, 0\right)=$ $\left(\bar{A}_{i}, 0\right)$ with $\bar{A}_{i}>0(i=1, \cdots, N)$, we still have the exact boundary controllability.

5). Since both the initial and final states belong to a piecewise $C^{1}$ neighbourhood of an arbitrarily given subcritical state, our results give only the local exact boundary controllability for unsteady flows in a tree-like network of open canals, however, that is enough to get the corresponding global exact boundary controllability. In fact, in order to drive any initial state given in a piecewise $C^{1}$ neighbourhood of a subcritical state to any final state given in a piecewise $C^{1}$ neighbourhood of another subcritical state, it suffices to use the previous results to drive the initial state to the first equilibrium state, then successively transfer the first equilibrium state to the second equilibrium state, and finally reach the final state. Thus we get the corresponding global exact boundary controllability, however, the controllability time must be enlarged many times.

Acknowledgments. LI Ta-Tsien is supported by the Special Funds for Major State Basic Research Projets of China. The paper was written during Rao Bopeng's visit in the Institut Sino-Français de Mathématiques Appliquées (ISFMA) at Fudan University on 2003-2004.

\section{REFERENCES}

[1] DE SAint-Venant, B., Théorie du mouvement non permanent des eaux, avec application aux crues des rivières et l'introduction des marées dans leur lit, C. R. Acad. Sci., 73 (1871), pp. $147-154,237-240$.

[2] Leugering, G. And Schmidt, E.G., On the modelling and stabilization of flows in networks of open canals, SIAM J. Control Optim., 41 (2002), pp. 164-180.

[3] Gugat, M. and Leugering, G., Global boundary controllability of the de St. Venant equations between steady states, Ann. I. H. Poincaré-AN20, 1 (2003), pp. 1-11.

[4] Coron,J.-M., D’Andréa-Novel, B. And Bastin, G., A Lyapunov approach to control irrigation canals modeled by Saint-Venant equations, in CD-Rom Proceedings, Paper F1008-5, ECC99, Karlsruhe, Germany, 1999.

[5] De Halleux, J., D'Andréa-Novel, B., Coron, J.-M. and Bastin, G., A Lyapunov approach for the control of multi reach channels modelled by Saint-Venant equations, in CD-Rom Proceedings, NOLCOS'01, St-Petersburg, Russia, June 2001, 1515-1520. 
[6] De Halleux, J. And Bastin, G., Stabilization of Saint-Venant equations using Riemann invariants: Application to waterways with mobile spillways, in CD-Rom Proceedings, Barcelona, Spain, July 2002.

[7] de Halleux, J., Prieur, C., Coron, J.-M., D’Andréa-Novel, B. and Bastin, G., Boundary feedback control in networks of open channels, Automatica, 39 (2003), pp. 1365-1376.

[8] Li T.T., Exact boundary controllability of unsteady flows in a network of open canals, Math. Nachr., 278 (2005), pp. 278-289.

[9] LI, T.T., Exact boundary controllability for quasilinear hyperbolic systems and its application to unsteady flows in a network of open canals, Math. Meth. Appl. Sci., 27 (2004), pp. $1089-1114$.

[10] LI, T.T. AND Jin, Y., Semi-global $C^{1}$ solution to the mixed initial-boundary value problem for quasilinear hyperbolic systems, Chin. Ann. Math., 22B (2001), pp. 325-336.

[11] Li, T.T., RaO, B.P. And Jin, Y., Solution $C^{1}$ semi-globale et contrôlabilité exacte frontière de systèmes hyperboliques quasi linéaires, C. R. Acad. Sci. Paris, t. 333, Série I (2001), pp. $219-224$.

[12] LI, T.T. AND RAO, B.P., Exact boundary controllability for quasilinear hyperbolic systems, SIAM J. Control Optim., 41 (2003), pp. 1748-1755.

[13] Li, T.T. AND RAO, B.P., Local exact boundary controllability for a class of quasilinear hyperbolic systems, Chin. Ann. Math., 23B (2002), pp. 209-218.

[14] LI T.T. AND YU L.X., Exact controllability for first order quasilinear hyperbolic systems with zero eigenvalues, Chin. Ann. Math., 24B (2003), pp. 415-422.

[15] Li T.T. And Yu W.C., Boundary Value Problems for Quasilinear Hyperbolic Systems, Duke University Mathematics Series V, 1985. 
T.-T. LI AND B. RAO 